\title{
Chip-Level Channel Equalization in WCDMA Downlink
}

\author{
Kari Hooli \\ Centre for Wireless Communications, P.O. Box 4500 FIN-90014, University of Oulu, Finland \\ Email: kari.hooli@ee.oulu.fi

\section{Markku Juntti} \\ Centre for Wireless Communications, P.O. Box 4500 FIN-90014, University of Oulu, Finland \\ Email: markku.juntti@ee.oulu.fi
}

\section{Markku J. Heikkilä}

Nokia Mobile Phones, P.O. Box 50, 90571 Oulu, Finland

Email: markku.j.heikkila@nokia.com

\section{Petri Komulainen}

Nokia Mobile Phones, P.O. Box 50, 90571 Oulu, Finland

Email:petri.komulainen@nokia.com

\section{Matti Latva-aho}

Centre for Wireless Communications, P.O. Box 4500 FIN-90014, University of Oulu, Finland Email: matla@ee.oulu.fi

\section{Jorma Lilleberg}

Nokia Mobile Phones, P.O. Box 50, 90571 Oulu, Finland

Email: jorma.lilleberg@nokia.com

Received 14 August 2001 and in revised form 7 March 2002

\begin{abstract}
The most important third generation (3G) cellular communications standard is based on wideband CDMA (WCDMA). Receivers based on TDMA style channel equalization at the chip level have been proposed for a WCDMA downlink employing long spreading sequences to ensure adequate performance even with a high number of active users. These receivers equalize the channel prior to despreading, thus restoring the orthogonality of users and resulting in multiple-access interference (MAI) suppression. In this paper, an overview of chip-level channel equalizers is delivered with special attention to adaptation methods suitable for the WCDMA downlink. Numerical examples on the equalizers' performance are given in Rayleigh fading frequency-selective channels.
\end{abstract}

Keywords and phrases: WCDMA, multiple-access interference, channel equalization.

\section{INTRODUCTION}

The air interface of universal terrestrial radio access (UTRA), the most important third generation (3G) cellular mobile communications standard, is based on wideband codedivision multiple-access (WCDMA). In 3G, cellular networks downlink capacity is expected to be more crucial than uplink capacity due to asymmetric capacity requirements, that is, the downlink should offer higher capacity than the uplink [1]. Therefore, the use of efficient downlink receivers is important. In order to avoid performance degradation, near-far resistant (or multiuser) receivers [2] can be used. Several suboptimal receivers feasible for practical implementations have been proposed, including linear minimum mean-squared error (LMMSE) receivers $[3,4]$. The adaptive versions of LMMSE receivers are the most promising for single-user terminals. However, the adaptive symbol-level LMMSE receivers rely on cyclostationarity of multiple access interference (MAI), and thus require periodic spreading sequences with a very short period. Hence, they cannot be applied to the frequency division duplex (FDD) mode of the WCDMA downlink, which uses spreading sequences with a one radio frame $(10 \mathrm{~ms})$ period. 
In a synchronously transmitted downlink employing orthogonal spreading codes, MAI is mainly caused by multipath propagation (neighboring cells form another source of MAI). Due to the nonzero cross-correlations between spreading sequences with arbitrary time shifts, there is interference between propagation paths (or Rake fingers) after despreading, causing MAI. With a moderate or high number of active users, the performance of a Rake receiver becomes limited by interpath MAI. If the received chip waveform, distorted by the multipath channel, is equalized prior to correlation by the spreading code or matched filtering, there is only a single path in the despreading. With orthogonal spreading sequences the equalization effectively retains, to some extent, the orthogonality of users lost due to multipath propagation, thus suppressing MAI. Since the signal is equalized at the chip level, not on the symbol level, they can also be applied in systems using long spreading sequences. Such a receiver consists of a linear equalizer followed by a single correlator and a decision device.

The chip-level channel equalizer has proven to be one of the most promising terminal receivers for a WCDMA/FDD downlink. It has drawn attention and inspired numerous publications in recent years. Chip-level equalization has been treated in $[5,6,7,8,9,10]$. It has been addressed in miscellaneous contexts also earlier, as in [11]. Although chiplevel equalization resembles at first glance the classical linear TDMA-type equalization (time-division multiple access), it has several aspects characteristic for CDMA or 3G networks, thus making it an interesting research topic. These aspects include efficient adaptation of the equalizer due to different training signals in CDMA, soft hand-over, and transmit diversity. Chip-level equalization was considered with soft hand-over in $[12,13]$, and with different transmit diversity schemes in $[14,15]$. Performance evaluations of chiplevel equalizers with channel coding have been presented in [16], and with adaptive equalizers in [17, 18]. A chip equalizer based receiver for a CDMA downlink employing a long scrambling sequence and nonorthogonal channelization sequences was proposed in [19]. A large variety of adaptive chip-level equalizers have also been presented and studied in a number of publications, including, for example, $[20,21,22,23,24,25,26,27]$. A different approach for improving the performance of downlink receiver, that is, generalized Rake receiver, is discussed in [28].

The purpose of this paper is to provide an overview of chip-level equalization and on the adaptation methods proposed for chip-level equalizers. The possible performance gains offered by the chip-level channel equalizers are addressed, and the performance of six different adaptive chip equalizers are compared in a frequency-selective fading channel. The paper is organized as follows. The system model is defined in Section 2, and the zero-forcing and LMMSE equalizers with perfect knowledge of the channel are addressed in Section 3. The adaptive versions of chip-level equalizers are discussed in Section 4. Numerical examples for the receivers are presented in Section 5, followed by concluding remarks in Section 6.

\section{SYSTEM MODEL}

In this section, we present the system model used in defining the receivers in the sequel. Only a single-base station and a single-receive antenna are included into the system model. ${ }^{1}$ With a single-receive antenna, signal processing is restricted to the time domain. Due to the lack of spatial characteristics in the signal structure, moderate level other cell interference can be considered to be included in the white Gaussian noise.

Since the downlink is considered, all signals are synchronously transmitted through the same multipath channel. The complex envelope of the received signal at the user terminal can be written as

$$
\begin{aligned}
r(t) & =\sum_{k=1}^{K} r_{k}(t)+n(t) \\
& =\sum_{k=1}^{K} \sum_{m=0}^{M_{k}-1} A_{k} b_{k}^{(m)} \sum_{l=1}^{L} c_{l}(t) s_{k}^{(m)}\left(t-m T_{k}-\tau_{l}\right)+n(t),
\end{aligned}
$$

where $K$ is the number of users, $M_{k}$ is the number of $k$ th user's symbols in the observation window, ${ }^{2} L$ is the number of paths, $A_{k}$ is the average received amplitude of $k$ th user, $b_{k}^{(m)}$ is the $m$ th symbol of $k$ th user, $c_{l}(t)$ is the time-variant complex channel coefficient of $l$ th path, $s_{k}^{(m)}(t)$ is the spreading waveform of $m$ th symbol of $k$ th user given by convolution of spreading sequence and chip waveform, $T_{k}$ is the symbol interval for $k$ th user, $\tau_{l}$ is the delay of $l$ th path, and $n(t)$ is complex white Gaussian noise process.

The discrete-time received signal after appropriate downconversion and filtering can be written as

$$
\mathbf{r}=\sum_{k=1}^{K} \mathbf{D C S}_{k} \mathbf{A}_{k} \mathbf{b}_{k}+\mathbf{n} \in \mathbb{C}^{N_{c} N_{s}}
$$

where $N_{s}$ is the number of samples per chip and $N_{c}$ is the number of chips in the observation window. ${ }^{3}$ In (2), $\mathbf{D}=$ $\left[\mathbf{d}_{1}^{(1)}, \ldots, \mathbf{d}_{L}^{(1)}, \mathbf{d}_{1}^{(2)}, \ldots, \mathbf{d}_{L}^{\left(N_{c}\right)}\right] \in \mathbb{R}^{N_{c} N_{s} \times L N_{c}}$ is a path delay and chip waveform matrix where the column vector $\mathbf{d}_{l}^{(n)}$ contains samples from appropriately delayed chip waveform for the $l$ th path of $n$th chip, $\mathbf{C}=\operatorname{diag}\left(\mathbf{c}^{(1)}, \ldots, \mathbf{c}^{\left(N_{c}\right)}\right) \in \mathbb{C}^{L N_{c} \times N_{c}}$ is a block diagonal channel matrix with column vector $\mathbf{c}^{(n)} \in \mathbb{C}^{L}$ containing the time-variant channel coefficients for $L$ paths. The term DC models the combination of chip waveform and multipath channel and is common for all users; $\boldsymbol{S}_{k}=$ $\operatorname{diag}\left(\mathbf{s}_{k}^{(1)}, \ldots, \mathbf{s}_{k}^{\left(M_{k}\right)}\right) \in \mathbb{C}^{N_{c} \times M_{k}}$ is a block diagonal spreading sequence matrix where column vector $s_{k}^{(m)} \in \Xi_{s}^{G_{k}}, \Xi_{s}$ is the chip alphabet, contains the spreading sequence for the $k$ th user's $m$ th symbol with a spreading factor $G_{k}$. The cell specific scrambling sequence is included in the spreading sequences, that is, $\mathbf{S}_{k}=\mathbf{S}_{\mathrm{sc}}(t) \mathbf{S}_{k, \mathrm{Ch}}$, where the scrambling

\footnotetext{
${ }^{1}$ It is straightforward to extend the model to include multiple-base stations or receive antennas.

${ }^{2}$ Thus the model allows multiple transmission rates.

${ }^{3} N_{c}=G_{k} M_{k}$, where $G_{k}$ is the spreading factor for $k$ th user. The product $G_{k} M_{k}$ is constant for all users.
} 
sequence is in the diagonal matrix $\mathbf{S}_{\mathrm{Sc}}(t)$ and the block diagonal matrix $\boldsymbol{S}_{k, \text { Ch }}$ contains the user specific channelization sequence for the $k$ th user. The sequences are normalized so that $\mathbf{S}_{k}^{\mathrm{H}} \boldsymbol{S}_{k}=\mathbf{I}$, and they are also orthogonal, that is, $\mathbf{S}_{k}^{\mathrm{H}} \mathbf{S}_{j}=\mathbf{0}$ if $k \neq j$. The average received amplitude for the $k$ th user is contained in a diagonal matrix $\mathbf{A}_{k}=A_{k} \mathbf{I}_{M_{k}}$, ${ }^{4}$ vector $\mathbf{b}_{k}=\left[b_{k}^{(1)}, \ldots, b_{k}^{\left(M_{k}\right)}\right]^{\mathrm{T}} \in \Xi_{b}^{M_{k}}, \Xi_{b}$ is the symbol alphabet, contains the transmitted symbols of $k$ th user, assumed to be i.i.d. with unit variance, and $\mathbf{n} \in \mathbb{C}^{N_{c} N_{s}}$ contains samples from a white complex Gaussian noise process with covariance $\mathbf{C}_{n n}=\sigma_{n}^{2} \mathbf{I}_{N_{c} N_{s}}$.

\section{RECEIVERS}

The chip-level version of zero-forcing and LMMSE equalizers as well as the conventional Rake receiver are presented in this section. As mentioned earlier, the multipath channel is equalized prior to despreading in the chip equalizer receivers, thus restoring to some extent the orthogonality of channelization codes and suppressing MAI. This means that the term DC in (2) is suppressed. The equalizer is followed by a single correlator and decision device.

The equalizers treated in this paper are all linear, due to the difficulties faced when nonlinear equalizers are considered for chip-level equalization. The detection of the desired user at chip-level is highly counter-intuitive, since it would ignore the processing gain associated with spreading. Thus, the desired output signal of a chip-level equalizer is the total transmitted signal from the base station, that is, the sum of all users' signals. The nonlinear equalizers rely on prior knowledge of constellation of the desired signal. This information is not easily available at the terminal, since the constellation of all users' signal sum is a high-order QAM with uneven spacing. The constellation order changes at frame rate $(100 \mathrm{~Hz})$ with the changing number of active users, and the spacing in the constellation changes at power control rate $(1.5 \mathrm{kHz})$. However, for example, in [29] an interesting nonlinear equalizer based on soft decision cancelling was presented for a WCDMA downlink.

With the introduced system model, the decision variable of the Rake receiver for arbitrary selected user 1 is given by

$$
\mathbf{y}_{R}=\mathbf{S}_{1}^{\mathrm{H}} \mathbf{C}^{\mathrm{H}} \mathbf{D}^{\mathrm{H}} \mathbf{r},
$$

that is, the received signal is filtered by the chip waveform, appropriately delayed and weighted with channel coefficients in the Rake fingers, coherently combined and finally despread. The abnormal order of the maximal ratio combining (MRC) and despreading has no effect on receiver performance due to the linearity of operations.

When a zero-forcing equalizer is applied, the tap coefficients of the equalizer are given by

$$
\mathbf{W}_{Z}=\mathbf{D C}\left(\mathbf{C}^{\mathrm{H}} \mathbf{D}^{\mathrm{H}} \mathbf{D C}\right)^{-1} .
$$

\footnotetext{
${ }^{4}$ The use of $\mathbf{A}_{k}$ allows power differences between different symbols of an individual user. In here, constant power $A_{k}^{2}$ is assumed.
}

The first term on the right-hand side, DC, performs chip waveform matched filtering, and is followed by the conventional zero-forcing equalizer $[30,31]$. The decision variable after despreading can be written as

$$
\mathbf{y}_{Z}=\mathbf{S}_{1}^{\mathrm{H}} \mathbf{W}_{Z}^{\mathrm{H}} \mathbf{r}=\mathbf{S}_{1}^{\mathrm{H}}\left(\mathbf{C}^{\mathrm{H}} \mathbf{D}^{\mathrm{H}} \mathbf{D C}\right)^{-1} \mathbf{C}^{\mathrm{H}} \mathbf{D}^{\mathrm{H}} \mathbf{r} .
$$

In (4), the zero-forcing equalizer is given in block form $\left(\mathbf{W}_{Z} \in \mathbb{C}^{N c N s \times N c}\right)$. The filter form of the zero-forcing equalizer is obtained by taking a middle column from $\mathbf{W}_{Z} \cdot{ }^{5}$

The second considered equalizer, an LMMSE chip-level equalizer, can be obtained by solving

$$
\mathbf{W}_{L}=\arg \min _{\mathbf{W}} \mathrm{E}\left[\left|\mathbf{W}^{\mathrm{H}} \mathbf{r}-\sum_{k=1}^{K} \mathbf{S}_{k} \mathbf{A}_{k} \mathbf{b}_{k}\right|^{2}\right],
$$

where $\mathrm{E}[\cdot]$ denotes expectations, and the minimization is carried out elementwise. By estimating the sum of all users' chips instead of the chips of a single user, the signal-to-noise ratio (SNR) faced in the estimation problem is significantly improved. It should also be noted that the chip equalizer does not try to suppress other users' signals at the chip level, but just restores the orthogonality of users. It can be shown that for (6) $\mathbf{W}_{L}$ is given by [32]

$$
\mathbf{W}_{L}=\left(\mathbf{D C}\left(\sum_{k=1}^{K} A_{k}^{2} \mathbf{S}_{k} \mathbf{S}_{k}^{\mathrm{H}}\right) \mathbf{C}^{\mathrm{H}} \mathbf{D}^{\mathrm{H}}+\sigma_{n}^{2} \mathbf{I}\right)^{-1} \mathbf{D C},
$$

resulting in the decision variable

$$
\begin{aligned}
\mathbf{y}_{L} & =\mathbf{S}_{1}^{\mathrm{H}} \mathbf{W}_{L}^{\mathrm{H}} \mathbf{r} \\
& =\mathbf{S}_{1}^{\mathrm{H}} \mathbf{C}^{\mathrm{H}} \mathbf{D}^{\mathrm{H}}\left(\mathbf{D C}\left(\sum_{k=1}^{K} A_{k}^{2} \mathbf{S}_{k} \mathbf{S}_{k}^{\mathrm{H}}\right) \mathbf{C}^{\mathrm{H}} \mathbf{D}^{\mathrm{H}}+\sigma_{n}^{2} \mathbf{I}\right)^{-1} \mathbf{r} .
\end{aligned}
$$

The standard symbol-level LMMSE equalizer [3, 4], feasible for DS-CDMA systems employing short spreading sequences, is defined for user 1 by

$$
\mathbf{W}_{L_{s}}=\arg \min _{\mathbf{W}} \mathrm{E}\left[\left|\mathbf{W}^{\mathrm{H}} \mathbf{r}-\mathbf{b}_{1}\right|^{2}\right],
$$

which results in the decision variable

$$
\mathbf{y}_{L_{s}}=\mathbf{A}_{1}^{\mathrm{H}} \mathbf{S}_{1}^{\mathrm{H}} \mathbf{C}^{\mathrm{H}} \mathbf{D}^{\mathrm{H}}\left(\mathbf{D C}\left(\sum_{k=1}^{K} A_{k}^{2} \mathbf{S}_{k} \mathbf{S}_{k}^{\mathrm{H}}\right) \mathbf{C}^{\mathrm{H}} \mathbf{D}^{\mathrm{H}}+\sigma_{n}^{2} \mathbf{I}\right)^{-1} \mathbf{r} .
$$

Comparing (8) and (10), we can see that, for a given observation window, the chip-level and symbol-level LMMSE receivers are equal up to a scalar. This is an expected result, since the LMMSE estimator commutes over linear (or affine) transformations [32], like despreading. The equal performance of LMMSE receivers was verified numerically in [33].

\footnotetext{
${ }^{5}$ In the sequel, filter forms are denoted with lower case $\mathbf{w}$.
} 
As seen from (7), the LMMSE solution of a chip-level equalizer depends on the spreading sequences of all users with the period of a long scrambling code. This follows from the dependency between consecutive chips to be estimated. The optimal solution changes from chip to chip, and an adaptive chip-level equalizer will not reach the exact optimal tap coefficients. The adaptive versions of chip-level LMMSE equalizer are built on simplifications that (i) spreading sequences are random, and (ii) the random spreading sequences are white and independent from those of other users. The simplifications are unavoidable due to the nonstationarity of the LMMSE solution in (7). ${ }^{6}$ The LMMSE chip-level equalizer derived according to the simplifications (or false assumptions) is given by

$$
\tilde{\mathbf{W}}_{L}=\left(s^{2} \sum_{k=1}^{K} A_{k}^{2} \mathbf{D} \mathbf{C} \mathbf{C}^{\mathrm{H}} \mathbf{D}^{\mathrm{H}}+\sigma_{n}^{2} \mathbf{I}\right)^{-1} \mathbf{D C},
$$

where $s^{2}$ denotes the square value of chip. The decision variable after correlation with the spreading sequence is given by

$$
\begin{aligned}
\mathbf{y}_{\tilde{L}} & =\mathbf{S}_{1}^{\mathrm{H}} \tilde{\mathbf{W}}_{L}^{\mathrm{H}} \mathbf{r} \\
& =\mathbf{S}_{1}^{\mathrm{H}} \mathbf{C}^{\mathrm{H}} \mathbf{D}^{\mathrm{H}}\left(s^{2} \sum_{k=1}^{K} A_{k}^{2} \mathbf{D C C} \mathbf{C}^{\mathrm{H}} \mathbf{D}^{\mathrm{H}}+\sigma_{n}^{2} \mathbf{I}\right)^{-1} \mathbf{r} .
\end{aligned}
$$

The performance losses caused by the aforementioned simplifications can be assessed by comparing the performances of the equalizers given in (7) and (11) [33, 34]. Again, the chip-level equalizer in (11) is in a block form, and the equalizer is obtained in filter form by taking a middle column from $\tilde{\mathbf{W}}_{L}$.

\section{ADAPTATION METHODS}

In this section, several methods for adapting chip-level equalizer are discussed. WCDMA terminal receivers have access to two-pilot signals, one carried on the continuous common pilot channel $(\mathrm{CPICH})$ and another carried on the time-multiplexed dedicated physical control channel (DPCCH). The high content of pilot signals combined with the DS-CDMA signal structure allows accurate channel response estimation in the terminal. On the other hand, pilot signals form only a small portion of the received signal. Thus, for the adaptation of the equalizer, a good estimate of the channel response and a relatively weak but time-continuous reference signal are available. As a result, several adaptation methods that are not typical with linear TDMA equalizers have been proposed for chip-level equalization. In [22] equalizing was proposed to be carried out by an adaptive chip separation filter, which is based on blindly decorrelating the multipath combined chip estimates. The method applies to systems employing long random scrambling codes, in which the original transmitted multiuser chip

\footnotetext{
${ }^{6}$ The LMMSE solution cannot be considered to be cyclostationary, since the channel is likely to change during the spreading sequence period of $10 \mathrm{~ms}$.
}

sequence is also uncorrelated. Kalman filtering was applied for chip-level equalization in [25]. Constrained minimum output energy equalizers, minimizing the energy in the subspace of unused spreading sequences, are proposed for IS-95 and CDMA2000 terminals in $[21,35,36,37]$. However, they require knowledge of unused spreading sequences, thus imposing restrictions too strict for radio resource management in WCDMA/FDD systems.

The most straightforward adaptation, that is, the least mean square (LMS) adaptation based on the common pilot channel (CPICH), is studied in Section 4.1. The use of a pilot channel as a reference signal was suggested in [7], but the adaptation was not studied any further. In [19], a pilot channel was proposed to be used with the stabilized fast aposteriori error sequential technique (SFAEST) and in [38] with the multistage nested Wiener filter (MSNWF). In the following, the adaptation is done at chip rate. The adaptation can be performed also at symbol rate, as shown in [39]. ${ }^{7}$ The use of Griffiths' algorithm [40] with a chip-level equalizer is presented in Section 4.2, followed by the introduction of a minimum output energy equalizer constrained with the channel response in Section 4.3. Equalizers consisting of a separate filter and Rake receiver are referred to as prefilterRake equalizers in the sequel $[20,27]$. In Section 4.4, adaptive versions of a prefilter-Rake based on square-root RLS, the Levinson algorithm and Griffiths algorithm are studied. Finally, the properties of adaptive equalizers are compared in Section 4.5.

\subsection{Common pilot channel based adaptation}

In the adaptive equalizers discussed in Sections 4.1, 4.2, and 4.3 , the appropriately sampled received signal is filtered by the chip waveform, equalized and correlated with the spreading sequence. The decision variable for user 1 after correlation with the spreading sequence is given by $\mathbf{y}=\mathbf{S}_{1}^{\mathrm{H}} \mathbf{z}$, where vector $\mathbf{z}$ contains equalizer outputs for corresponding chip intervals. The $n$th element of $\mathbf{z}$ is $\mathbf{w}(n)^{\mathrm{H}} \overline{\mathbf{r}}(n)$, where $\mathbf{w}(n) \in$ $\mathbb{C}^{2 D+1}$ contains the equalizer taps and $\overline{\mathbf{r}}(n)=\left[r\left(n N_{s}-D\right)\right.$, $\left.\ldots, r\left(n N_{s}\right), \ldots, r\left(n N_{s}+D\right)\right]^{\mathrm{T}}$ contains output samples from the chip waveform matched filter within the equalizer at $n$th chip interval. The number of samples per chip is given by $N_{s}$ and $2 D+1$ is the number of equalizer taps.

The most straightforward solution to the adaptation of a chip-level equalizer is to use the normalized LMS (NLMS) algorithm with a common (or dedicated) pilot channel as a reference signal. The receiver is depicted in Figure 1, and referred to as a CPICH trained equalizer in the following. The NLMS adaptation step for the equalizer is [41, Chapter 9]

$$
\mathbf{w}_{C}(n+1)=\mathbf{w}_{C}(n)+\mu \frac{\overline{\mathbf{r}}(n) e^{*}(n)}{\overline{\mathbf{r}}^{\mathrm{H}}(n) \overline{\mathbf{r}}(n)},
$$

where $0<\mu<2$ is the adaptation step size, and $e^{*}(n)=$ $\left(s(n)-\mathbf{w}_{C}^{\mathrm{H}}(n) \overline{\mathbf{r}}(n)\right)^{*}$ is the complex conjugate of error signal between the equalizer output and reference signal $s(n)$.

\footnotetext{
${ }^{7}$ When comparing results, one should pay attention to the channel model assumptions made for the fractionally spaced equalizer.
} 


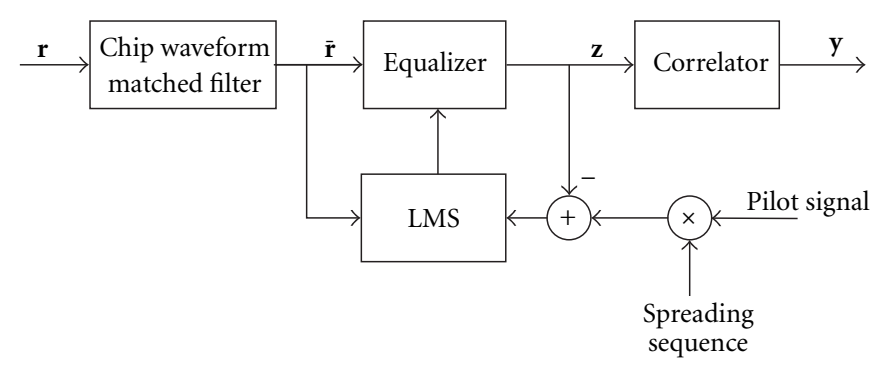

Figure 1: CPICH trained LMS equalizer.

Since the adaptation is carried out at chip level, the chips of CPICH are used as reference samples. It should be noted that although the error $e(n)$ contains the signals of all active users, also the desired one, the equalizer does not suppress them due to the pseudorandomness of spreading codes. The relatively large error signal values also mean that the SNR in the adaptation is low, and small adaptation step sizes are required to provide sufficient averaging. The small values of $\mu$ are partially compensated by a high adaptation rate.

\subsection{Griffiths' algorithm adapted equalizer}

Several adaptation algorithms are obtained through different approximations of the gradient vector of the mean square error cost function

$$
J=\mathrm{E}\left[\left|d-\mathbf{w}^{\mathrm{H}} \overline{\mathbf{r}}\right|^{2}\right],
$$

where $d$ is the desired output of the equalizer. The gradient vector is given by

$$
\nabla J=-2 \mathrm{E}\left[d^{*} \overline{\mathbf{r}}\right]+2 \mathrm{E}\left[\overline{\mathbf{r}}^{\mathrm{H}}\right] \mathbf{w},
$$

where $\mathrm{E}\left[d^{*} \overline{\mathbf{r}}\right]$ is the cross-correlation vector between the input signal $\overline{\mathbf{r}}$ and the desired output of the equalizer, and $\mathrm{E}\left[\overline{\mathbf{r}} \overline{\mathbf{r}}^{\mathrm{H}}\right]=\mathbf{R}$ is the covariance matrix of the input signal [41]. For example, the aforementioned standard LMS algorithm is obtained by replacing expectations with instantaneous estimates, that is, signal vectors $\overline{\mathbf{r}}(n)$.

In [23], the Griffiths algorithm is used for the adaptation of a chip-level channel equalizer. The algorithm is obtained from (15) by replacing the cross-correlation vector $\mathrm{E}\left[d^{*} \overline{\mathbf{r}}\right]$ with $\hat{\mathbf{p}}$, the channel response estimate. Instantaneous estimates are used for the covariance matrix $\mathbf{R}$, as in the LMS algorithm. The resulting adaptation is

$$
\mathbf{w}_{G}(n+1)=\mathbf{w}_{G}(n)-\mu\left(z^{*}(n) \overline{\mathbf{r}}(n)-\hat{\mathbf{p}}\right),
$$

where $\mu$ is the adaptation step size and $z(n)$ is the equalizer output at $n$th chip interval.

\subsection{CR-MOE equalizer}

In the channel-response constrained minimum-outputenergy (CR-MOE) equalizer [26], the equalizer is decomposed into a constraint (or nonadaptive) component and an adaptive component. This is the well-known idea of the gen- eralized side-lobe canceler, described, for example, in [41, Chapter 5]. The same approach has been applied in blind MOE multiuser receivers, in which the spreading sequence of a desired user is used as the constraint [2, 42]. As mentioned, the equalizer is decomposed into two parts, that is, $\mathbf{w}_{M}=\hat{\mathbf{p}}+\mathbf{x}$. The channel response estimate $\hat{\mathbf{p}}$ is used as the nonadaptive part, and the adaptive part $\mathbf{x}$ is constrained onto a subspace orthogonal to $\hat{\mathbf{p}}$ to avoid suppression of the desired signal. The mean square error $J=\mathrm{E}\left|d-\mathbf{w}_{M}^{\mathrm{H}} \mathbf{r}\right|^{2}$ can be written as $J=\mathrm{E}\left[d^{2}\right]-2 \hat{\mathbf{p}}^{\mathrm{H}} \hat{\mathbf{p}}+(\hat{\mathbf{p}}+\mathbf{x})^{\mathrm{H}} \mathrm{E}\left[\overline{\mathbf{r}} \overline{\mathbf{r}}^{\mathrm{H}}\right](\hat{\mathbf{p}}+\mathbf{x})$. For a given $\hat{\mathbf{p}}$, the mean square error is minimized by minimizing the last term of $J$, that is, the equalizer output energyother terms of $J$ are not affected by vector $\mathbf{x}$. To obtain an adaptive algorithm for $\mathbf{x}$, stochastic approximation is applied to the gradient $\nabla J=\mathrm{E}\left[\overline{\mathbf{r}}^{\mathrm{H}}\right](\hat{\mathbf{p}}+\mathbf{x})$ taken with respect to $\mathbf{x}$. The orthogonality condition is maintained at each iteration by projecting the gradient onto the subspace orthogonal to $\hat{\mathbf{p}}$. The orthogonal component of the gradient is

$$
\nabla \tilde{J}_{\perp \hat{\mathbf{p}}}=\left(\overline{\mathbf{r}}-\hat{\mathbf{p}} \frac{\hat{\mathbf{p}}^{\mathrm{H}} \overline{\mathbf{r}}}{\hat{\mathbf{p}}^{\mathrm{H}} \hat{\mathbf{p}}}\right) \overline{\mathbf{r}}^{\mathrm{H}} \mathbf{w}_{M}
$$

and the resulting adaptation algorithm becomes

$$
\mathbf{x}(n+1)=\mathbf{x}(n)-\mu z^{*}(n)\left(\overline{\mathbf{r}}(n)-z_{p}(n) \hat{\mathbf{p}}\right),
$$

where $z_{p}(n)=\hat{\mathbf{p}}^{\mathrm{H}} \overline{\mathbf{r}}(n) /\left(\hat{\mathbf{p}}^{\mathrm{H}} \hat{\mathbf{p}}\right)$ is the output of the channel response filter normalized with the energy of the channel response estimate, and $z(n)=\mathbf{w}_{M}(n)^{\mathrm{H}} \overline{\mathbf{r}}=(\hat{\mathbf{p}}(n)+\mathbf{x}(n))^{\mathrm{H}} \overline{\mathbf{r}}$ is the output of the CR-MOE equalizer. The structure of this equalizer is depicted in Figure 2.

The CR-MOE has the typical weaknesses of the MOE adaptation [2]. The orthogonality between $\mathbf{x}$ and the channel response estimate $\hat{\mathbf{p}}$ is lost when $\hat{\mathbf{p}}$ is updated. Thus periodic re-orthogonalization of $\mathbf{x}$ is required, given by

$$
\mathbf{x}_{\perp \hat{\mathbf{p}}}=\mathbf{x}-\frac{\hat{\mathbf{p}}^{\mathrm{H}} \mathbf{x}}{\hat{\mathbf{p}}^{\mathrm{H}} \hat{\mathbf{p}}} \hat{\mathbf{p}}
$$

The second problem of the MOE adaptation is the unavoidable estimation error in $\hat{\mathbf{p}}$. Due to the estimation error, $\mathbf{x}$ has small projection on true $\mathbf{p}$ while maintaining orthogonality with $\hat{\mathbf{p}}$. Since $\mathbf{x}$ is adapted to minimize output energy, the projection on $\mathbf{p}$ translates to partial suppression of the desired signal component. The surplus energy $\chi=\|\mathbf{x}\|^{2}$, where $\|\mathbf{x}\|^{2}=\mathbf{x}^{\mathrm{H}} \mathbf{x}$, required for total suppression of the desired signal component is given by

$$
\chi \geq \frac{\|\hat{\mathbf{p}}\|^{2}\left|\hat{\mathbf{p}}^{\mathrm{H}} \mathbf{p}\right|^{2}}{\|\hat{\mathbf{p}}\|^{2}\|\mathbf{p}\|^{2}-\left|\hat{\mathbf{p}}^{\mathrm{H}} \mathbf{p}\right|^{2}} .
$$

Since the channel estimation error is usually relatively small, $\left(\left|\hat{\mathbf{p}}^{\mathrm{H}} \mathbf{p}\right|^{2} \sim\|\hat{\mathbf{p}}\|^{2}\|\mathbf{p}\|^{2}\right)$, suppression of the desired signal means large $\|\mathbf{x}\|^{2}$ values and significant noise enhancement. Therefore, in noisy environments the suppression remains at acceptable levels. However, to avoid the desired signal suppression at high SNR, $\|\mathbf{x}\|^{2}$ values must be restricted. One solution is to introduce tap leakage [2], that is, 


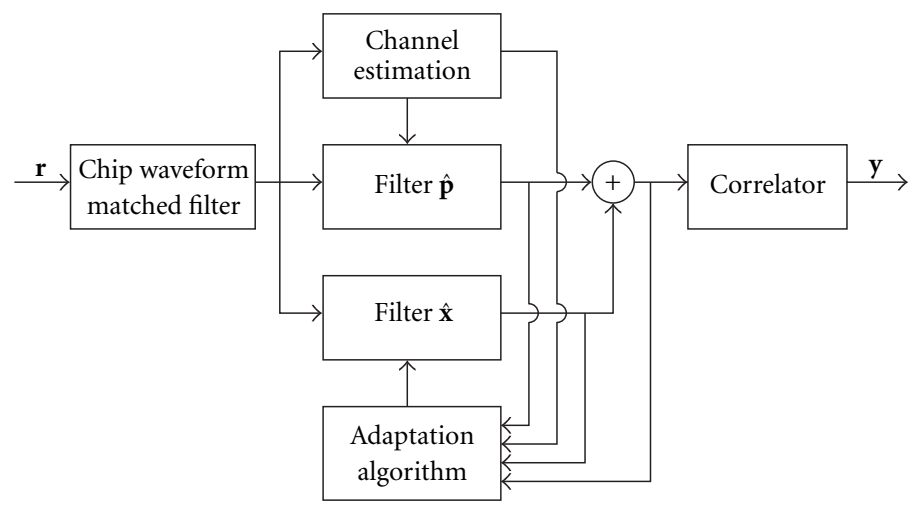

FIGURE 2: Channel-response constrained minimum-output-energy (CR-MOE) equalizer.

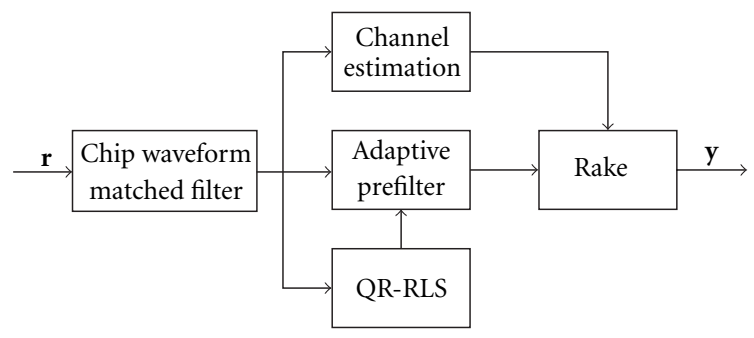

FIgURE 3: Prefilter-Rake receiver with inverse QR-RLS adaptation.

$$
\mathbf{x}(n+1)=(1-\mu \alpha) \mathbf{x}(n)-\mu z^{*}(n)\left(\overline{\mathbf{r}}(n)-z_{p}(n) \hat{\mathbf{p}}\right),
$$

where $\alpha$, a small positive constant, controls the tap leakage. On the other hand, large values of $\alpha$ cause too large tap leakage, thus preventing efficient channel equalization. However, a predefined constant $\alpha$ has proven to be adequate, if $\hat{\mathbf{p}}$ is normalized with its energy.

\subsection{Prefilter-Rake equalizer}

When (3) and (12) are compared, it can be seen that the equalizer consists of a received signal's covariance matrix inverse, and of the part corresponding to the conventional Rake receiver. In [20] it was suggested to actually divide the equalizer into these parts, and to use the matrix inversion lemma (or appropriate parts of the RLS-algorithm) for the estimation of covariance matrix inverse $\mathbf{R}^{-1}\left(\mathbf{R}=\mathrm{E}\left[\overline{\mathbf{r}} \overline{\mathbf{r}}^{\mathrm{H}}\right]\right)$. If the chip waveform matched filter is sampled at chip rate, the covariance matrix has a Toeplitz structure. Also the matrix inverse $\mathbf{R}^{-1}$ approaches a Toeplitz matrix with increasing matrix dimension and finite effective length of the autocorrelation function. Thus the multiplication with $\mathbf{R}^{-1}$ can be effectively replaced by filtering $\overline{\mathbf{r}}$ with a middle row of $\mathbf{R}^{-1}$.

The structure of prefilter-Rake with channel estimation is depicted in Figure 3. A filter matched to the chip waveform is preceding the prefilter, and the output of the matched filter is sampled at chip rate. The output of the prefilter is fed to the Rake receiver performing despreading and maximal ratio combining. There exist several possibilities for adapting the prefilter. In the following, three methods are presented. First, a blind approach utilizing square-root RLS algorithms [27] is discussed, followed by a description of adaptation using the Levinson algorithm. The use of the Levinson algorithm was proposed also in the simultaneous and independent work of Mailaender [43]. Finally, the application of Griffiths' algorithm to the prefilter adaptation [18] is discussed.

\section{Square-root $R L S$}

The coefficients of the prefilter $\mathbf{v}(n)$ are given by the middle column of $\hat{\mathbf{R}}^{-1}(n),{ }^{8}$ estimate of covariance matrix inverse $\mathbf{R}^{-1}$. The square-root matrix of $\hat{\mathbf{R}}^{-1}(n)$ can be updated using appropriate parts of the (inverse) QR-RLS algorithm [41, Chapter 14], or as in this paper, Householder RLS (HRLS) [44]. Both algorithms operate on the squareroot matrix $\hat{\mathbf{R}}^{1 / 2}(n)$ of $\hat{\mathbf{R}}^{-1}$, that is, $\hat{\mathbf{R}}^{-1}(n)=\hat{\mathbf{R}}^{1 / 2}(n) \hat{\mathbf{R}}^{\mathrm{H} / 2}(n)$. Thus the prefilter coefficients are obtained by $\mathbf{v}(n)=$ $\hat{\mathbf{R}}^{1 / 2}(n)\left[\hat{R}^{H / 2}(n)\right]_{:, D+1}$, where $\left[\hat{R}^{H / 2}(n)\right]_{:, D+1}$ denotes the middle column of $\hat{\mathbf{R}}^{\mathrm{H} / 2}(n)$. The square-root matrix is restricted to a triangular matrix in the QR-RLS algorithm, whereas in the HRLS algorithm there is no such restriction. This allows an efficient use of block annihilation properties of Householder reflection. The HRLS update of $\hat{\mathbf{R}}^{\mathrm{H} / 2}(n)$ can be written as [44]

$$
\begin{aligned}
\Theta(n)\left[\begin{array}{cc}
\beta^{-1 / 2} \hat{\mathbf{R}}^{\mathrm{H} / 2}(n) & \beta^{-1 / 2} \hat{\mathbf{R}}^{\mathrm{H} / 2}(n) \overline{\mathbf{r}}(n) \\
\mathbf{0}^{\mathrm{T}} & 1
\end{array}\right] \\
=\left[\begin{array}{cc}
\hat{\mathbf{R}}^{\mathrm{H} / 2}(n+1) & \mathbf{0} \\
\mathbf{k}^{\mathrm{T}}(n) & -\delta(n)
\end{array}\right],
\end{aligned}
$$

where $0<\beta<1$ is a weighting factor and $\hat{\mathbf{R}}^{\mathrm{H} / 2}(n) \overline{\mathbf{r}}(n)$ is a preprocessed input vector; $\Theta(n)$ is a Householder transformation matrix annihilating $\hat{\mathbf{R}}^{\mathrm{H} / 2}(n) \overline{\mathbf{r}}(n)$ to a zero vector in the postarray. The resulting adaptation algorithm for the prefilter coefficients is tabulated in Table 1. It should be noted that prefilter-Rake does not require computation of $\delta(n)$ or $\mathbf{k}(n)$ in the postarray. Although the square-root RLS

\footnotetext{
${ }^{8}$ The filtering with a prefilter is defined as $\mathbf{v}^{\mathrm{H}}(n) \overline{\mathbf{r}}(n)$.
} 
TABle 1: Prefilter adaptation with Householder RLS algorithm.

At every prefilter update

$\mathbf{A}=\beta^{-1 / 2} \hat{\mathbf{R}}^{\mathrm{H} / 2}(n)$

$\mathbf{a}=\mathbf{A} \overline{\mathbf{r}}(n)$

$\mathbf{B}=\mathbf{A}^{\mathrm{H}} \mathbf{a}$

$\gamma=\sqrt{\mathbf{a}^{\mathrm{H}} \mathbf{a}+1}$

$\gamma=[\gamma(\gamma+1)]^{-1}$

$\mathbf{B}=\gamma \mathbf{B}$

$\hat{\mathbf{R}}^{\mathrm{H} / 2}(n+1)=\mathbf{A}-\mathbf{a B}^{\mathrm{H}}$

$\mathbf{v}(n+1)=\hat{\mathbf{R}}^{1 / 2}(n+1)\left[\hat{\mathbf{R}}^{\mathrm{H} / 2}(n+1)\right]_{:, D+1} \quad$ Prefilter length is $2 D+1$

algorithms are computationally intensive, the filter lengths in the chip-level equalizers are relatively short, roughly twice the effective channel delay spread.

\section{Levinson algorithm}

Since the ideal prefilter coefficients $\mathbf{v}(n)$ are given by a middle column of matrix inverse $\mathbf{R}^{-1}$, the prefilter coefficients can be obtained by solving periodically

$$
\hat{\mathbf{R}}(n) \mathbf{v}(n)=[\mathbf{I}]_{: D+1},
$$

where $[\mathbf{I}]_{:, D+1}$ is the middle column of the identity matrix, where $\mathbf{I}$ is the identity matrix, and $2 D+1$ is the prefilter length. Due to the Toeplitz structure of $\mathbf{R},(23)$ is efficiently solved with the standard Levinson algorithm, tabulated, for example, in [45]. Since the covariance matrix $\mathbf{R}$ is Toeplitz and Hermitean, it is defined by its first row. Thus, it is sufficient to estimate the autocorrelation function $\rho$ of $\overline{\mathbf{r}}$ for nonnegative delays.

Estimation of autocorrelation function can be done either directly from the received signal [32], or the estimate $\hat{\rho}$ can be calculated from the channel response estimate $\hat{\mathbf{p}}$ and noise power estimate $\hat{\sigma}_{n}^{2}$ by

$$
\rho[k]=\hat{\mathbf{p}}_{1: 2 D+1-k}^{\mathrm{H}} \hat{\mathbf{p}}_{1+k: 2 D+1}+\delta_{k} \hat{\sigma}_{n}^{2}
$$

for lags $k=0, \ldots, 2 D$. Calculation of the autocorrelation estimate from the channel response estimate has lower complexity than the aforementioned possibilities, since the channel response estimate is required in other parts of receiver and the autocorrelation estimate can be updated at the prefilter update rate. However, it is less robust against any unknown interfering signals.

To maintain good performance, the prefilter coefficients should be recalculated several times during the coherence time of the channel. Thus the update frequency can be fixed, for example, to the WCDMA slot rate $(1.5 \mathrm{kHz})$, or can be made to adapt to the velocity of the terminal. To avoid any performance degradations due to a compromise with the prefilter update rate, a $15 \mathrm{kHz}$ update rate is used in the simulation cases presented in Section 5.

\section{Modified Griffiths' algorithm}

The ideas of Griffiths' algorithm can be used to deriving an LMS algorithm variant for the prefilter adaptation [18]. The gradient of mean square error cost function for the prefilter $\mathbf{v}$ is

$$
\nabla J=-2 \mathrm{E}\left[d^{*} \overline{\mathbf{r}}\right]+2 \mathrm{E}\left[\overline{\mathbf{r}} \overline{\mathbf{r}}^{\mathrm{H}}\right] \mathbf{v}
$$

The desired response of the prefilter is the received signal filtered with the desired prefilter, that is, $d=\left[\mathbf{R}^{-1}\right]_{:, D+1}^{\mathrm{H}} \overline{\mathbf{r}}$. This allows a further development of the cost function gradient

$$
\begin{aligned}
\nabla J & =-2 \mathrm{E}\left[\overline{\mathbf{r}} \overline{\mathbf{r}}^{\mathrm{H}}\right]\left[\mathbf{R}^{-1}\right]_{: D+1}+2 \mathrm{E}\left[\overline{\mathbf{r}} \overline{\mathbf{r}}^{\mathrm{H}}\right] \mathbf{v} \\
& =-2[\mathbf{I}]_{:, D+1}+2 \mathrm{E}\left[\overline{\mathbf{r}} \overline{\mathbf{r}}^{\mathrm{H}}\right] \mathbf{v}
\end{aligned}
$$

The adaptive algorithm is obtained by replacing the remaining expectation with an instantaneous estimate of expectation, that is, with $\overline{\mathbf{r}} \overline{\mathbf{r}}^{\mathrm{H}}$. The resulting adaptation algorithm for the prefilter can be written as

$$
\mathbf{v}(n+1)=\mathbf{v}(n)+\mu\left([\mathbf{I}]_{:, D+1}-g^{*}(n) \overline{\mathbf{r}}(n)\right),
$$

where $g(n)=\mathbf{v}(n)^{\mathrm{H}} \overline{\mathbf{r}}(n)$ is the output of the prefilter.

Since the inverse of a Hermitean Toeplitz matrix is Hermitean and persymmetric [46], the optimal prefilter $\left[\mathbf{R}^{-1}\right]_{:, D+1}$ is conjugate symmetric with respect to its middle element. This can be utilized to speed up the adaptation by forcing

$$
\begin{aligned}
& \overline{\mathbf{v}}_{D-m}(n)=\frac{1}{2}\left(\mathbf{v}_{D-m}(n)+\mathbf{v}_{D+m}^{*}(n)\right), \\
& \overline{\mathbf{v}}_{D+m}(n)=\overline{\mathbf{v}}_{D-m}^{*}(n), \quad m=0,1, \ldots, D
\end{aligned}
$$

at each adaptation step and by using filter $\overline{\mathbf{v}}(n)$ instead of $\mathbf{v}(n)$ to generate the new prefilter output.

\subsection{Observations on adaptive equalizers}

In this section, the similarities and differences between the studied equalizers are briefly discussed. Firstly, it should be noted that the computational requirements of the presented equalizers are quite similar, with the exception of prefilterRake with square-root RLS adaptation. The complexity of the prefilter-Rake with RLS-type adaptation increases as a quadratic function of the number of taps, whereas the complexities of the other equalizers depend roughly linearly on the number of taps. Thus, the complexity of the squareroot RLS adapted prefilter-Rake is considerably higher than the complexities of the other presented equalizers. Also the Levinson algorithm has quadratic complexity, but the higher complexity is effectively compensated for by the significantly lower activation rate of the Levinson algorithm.

A CPICH trained equalizer treats other received signals than CPICH signal as noise, whereas all other equalizers utilize the whole received signal from the desired base station in the adaptation. The use of the whole received signal significantly enhances the SNR available in the equalizer adaptation, thus providing an advantage for the other equalizers over the CPICH trained equalizer.

On the other hand, a CPICH trained equalizer does not require channel response estimate, whereas the other equalizers rely on channel estimation. In prefilter-Rake receivers 
TABLE 2: Channel parameters.

\begin{tabular}{c|cccc}
\hline & \multicolumn{2}{|c}{ Vehicular A } & \multicolumn{2}{c}{ Channel II } \\
\hline Path \# & Power [dB] & Delay [ns] & Power [dB] & Delay [ns] \\
1 & 0 & 0 & 0 & 0 \\
2 & -1 & 310 & -3 & 521 \\
3 & -9 & 710 & -6 & 1042 \\
4 & -10 & 1090 & - & - \\
5 & -15 & 1730 & - & - \\
6 & -20 & 2510 & - & - \\
\hline
\end{tabular}

channel estimates are needed for maximal ratio combining, but the adaptation of the prefilter can be carried out blindly. In the CR-MOE and Griffiths' equalizers, MAI suppression is based on channel response estimate. With the CR-MOE equalizer an inaccurate channel response estimate also results in self-cancellation. Thus the CR-MOE receiver can be considered to be the most sensitive to channel estimation errors of the considered equalizers.

It can be easily noted that the CR-MOE and Griffiths' equalizers have a distinctive resemblance. For example, the part of the adaptation step orthogonal to $\hat{\mathbf{p}}$ in (16) is equal to the adaptation step in (18), assuming the same equalizer taps $\mathbf{w}(n)$. However, the estimated channel response is directly inserted into the equalizer in CR-MOE, whereas in Griffiths' algorithm it is gradually introduced through the adaptation. This provides a small advantage in the tracking of the changing channel.

Finally, it should be noticed that the prefilter-Rake structure offers an additional advantage in the soft hand-over situation. Since the prefilter depends only on the properties of received signal, the same prefilter can be used in the detection of signals coming from different base stations. In the soft hand-over situation, the prefilter is followed by more than one Rake receiver, each of them assigned to a different base station.

\section{NUMERICAL EXAMPLES}

To obtain a good understanding and comparison of the potential performance that the presented receivers can offer, bit error probabilities (BEPs) and signal-to-interference-plusnoise ratios (SINRs) were evaluated for the Rake receiver, zero-forcing equalizer and LMMSE equalizers with ideal coefficients. Bit error rates (BERs) were simulated for the presented adaptive chip-level equalizers.

The performance evaluations were carried out in Rayleigh fading frequency-selective channels described in Table 2. The ITUs vehicular A channel model [47] was used with BEP and SINR evaluations. The BER simulations were carried out in Channel II, which is a 3-path channel with an exponentially decaying average power profile. QPSK modulation was used employing root raised cosine pulses with a roll-off factor of 0.22. Random cell specific scrambling and Walsh channelization codes were used. The chip rate was set to $3.84 \mathrm{Mchip} / \mathrm{s}$ corresponding to a $260 \mathrm{~ns}$ chip interval. The received signal was modeled with two samples per chip, and channel coding was excluded from the study.

\subsection{Bit error probability and signal-to-interference-plus-noise evaluations}

BEPs were evaluated by applying the semianalytical method discussed, for example, in [48]. For a linear receiver $\mathbf{w},{ }^{9}$ the conditional bit error probability can be expressed in the form ${ }^{10}$

$$
\begin{aligned}
P_{b}\left[\mathrm{E} \mid \mathbf{C}, \mathbf{b}_{1}, \ldots, \mathbf{b}_{K}\right] & \\
= & Q\left(\frac{\operatorname{Re}\left(\sum_{k=1}^{K} \mathbf{w}^{\mathrm{H}} \mathbf{D C} \mathbf{S}_{k} \mathbf{A}_{k} \mathbf{b}_{k}\right)}{\sqrt{\sigma_{n}^{2} \mathbf{w}^{\mathrm{H}} \mathbf{w}}}\right),
\end{aligned}
$$

given that the arbitrary selected desired user 1 transmitted QPSK symbol 1. The bit error probability is conditional on channel realization, on the symbols of other users as well as on the previous and following symbols of the desired user. The BEPs were obtained by taking the average of the conditional bit error probabilities over a sample set of bit pattern and channel realizations. A sample set size of 8000 realizations was used in the evaluations.

In the same manner SINR was also evaluated. The conditional SINR depending on channel realization and bit patterns is given by

$$
\gamma=\frac{\operatorname{Re}\left(\mathbf{w}^{\mathrm{H}} \mathbf{D C S} \mathbf{S}_{1} \mathbf{A}_{1} \mathbf{b}_{1}\right)^{2}}{\sigma_{n}^{2} \mathbf{w}^{\mathrm{H}} \mathbf{w}+\left|\sum_{k=2}^{K} \mathbf{w}^{\mathrm{H}} \mathbf{D C S} \mathbf{S}_{k} \mathbf{A}_{k} \mathbf{b}_{k}\right|^{2}+\operatorname{Im}\left(\mathbf{w}^{\mathrm{H}} \mathbf{D C S} \mathbf{S}_{1} \mathbf{A}_{1} \mathbf{b}_{1}\right)^{2}},
$$

and a Gaussian approximation for the conditional bit error probability is obtained by

$$
\tilde{P}_{b}\left[\mathrm{E} \mid \mathbf{C}, \mathbf{b}_{1}, \ldots, \mathbf{b}_{K}\right]=Q(\sqrt{\gamma}) .
$$

Both the conditional SINR and the Gaussian approximation for conditional bit error probability were sampled over a sample set of bit pattern and channel realizations.

BEPs given by (29) and (31) were evaluated for 8 users employing a spreading factor of 16 and equal transmission powers in the vehicular A channel. The length of zero-forcing and LMMSE equalizers was set to 98 taps ( 49 chips), and the observation window of the received signal was 80 chips. BEPs are presented in Figure 4 for the conventional Rake receiver, as well as for the ZF and LMMSE chip-level equalizers $\mathbf{W}_{L}$ and $\tilde{\mathbf{W}}_{L}$ given by (7) and (11), respectively. Also the theoretical single-user bound [49] for the considered channel is given in the figure.

From the results in Figure 4 it can be immediately noted that the Gaussian approximation for BEP gives a good match with the BEP values obtained with (29). Also it is easily seen that as $E_{b} / N_{0}$ increases, the $\mathrm{BEP}$ of the Rake receiver saturates due to the MAI. The LMMSE equalizers show a significant BEP improvement when compared to conventional Rake

\footnotetext{
${ }^{9}$ Correlation with a spreading code is included in $\mathbf{w}$.

${ }^{10} \mathrm{Gray}$ code mapping of symbols to bits is assumed, and the term $\mathrm{Q}^{2}(\cdot)$ is ignored.
} 


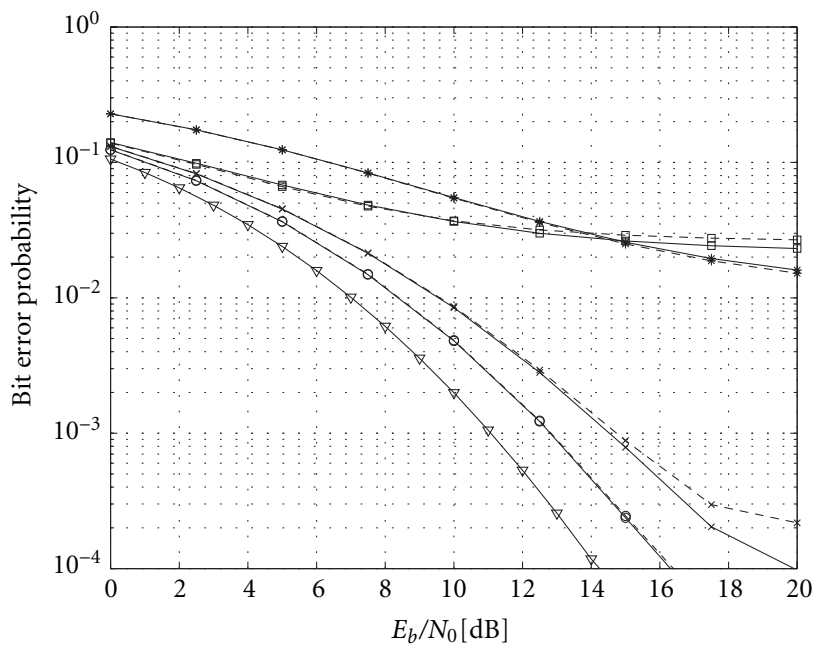

FIgURE 4: BEP versus $E_{b} / N_{0}$ for 8 QPSK users with a spreading factor of 16 in the Vehicular A channel. BEP (solid line) are presented with Gaussian approximation (dashed line) for Rake receiver $(\square)$, zero-forcing equalizer $(*)$, as well as for the LMMSE receivers given by $(7)(\circ)$ and by $(11)(\times)$. Also the performance bound of channel $(\nabla)$ is included.

receiver, whereas the $\mathrm{ZF}$ equalizer attains the performance of a Rake receiver only at high $E_{b} / N_{0}$. The performance difference between ZF and LMMSE chip-level equalizer receivers is caused by the noise enhancement typical to the ZF equalizers [50]. The performance difference between the LMMSE equalizers $\mathbf{W}_{L}$ and $\tilde{\mathbf{W}}_{L}$ is relatively small, less than $2 \mathrm{~dB}$ at the practical $E_{b} / N_{0}$ range. Hence, it can be noted that the changing chip correlations do not have a significant effect on the performance of the LMMSE equalizer in a WCDMA downlink. However, the LMMSE equalizer defined by (11) shows moderate saturation in performance at high $E_{b} / N_{0}$ values.

In Figure 5, the sample distribution functions are presented for the SINR $\gamma$ with the Rake receiver, the ZF equalizer and LMMSE equalizers. The sample distribution functions are given for $E_{S} / N_{0}$ values of $6 \mathrm{~dB}$ and $18 \mathrm{~dB}$. The SINR for a zero-forcing equalizer presents a strongly skewed sample distribution function with a heavily weighted lower tail. Comparison of Figures 4 and 5 reveals that the BEP performance is dominated by the lower tail characteristics of an SINR distribution, as expected. At $E_{b} / N_{0}=15 \mathrm{~dB}$, the $\mathrm{BEP}$ of the $\mathrm{ZF}$ equalizer is close to that of the Rake receiver. At the corresponding $E_{S} / N_{0}(18 \mathrm{~dB})$, the median SINR of the ZF equalizer is closer to the median SINR of $\tilde{\mathbf{W}}_{L}$, whereas the 10th percentiles ${ }^{11}$ of SINR are almost equal for the ZF equalizer and Rake receiver. From Figure 5 we can also notice that with increasing $E_{S} / N_{0}$ the variance of SINR increases significantly more for the Rake receiver than for LMMSE equalizers. Thus LMMSE equalizers provide a more stable SINR performance than the Rake receiver, which is an important advantage for radio resource management processes.

\footnotetext{
${ }^{11} 10$ th percentile of a sample set is such a value $x$ that at least $10 \%$ of samples are smaller than or equal to $x$.
}

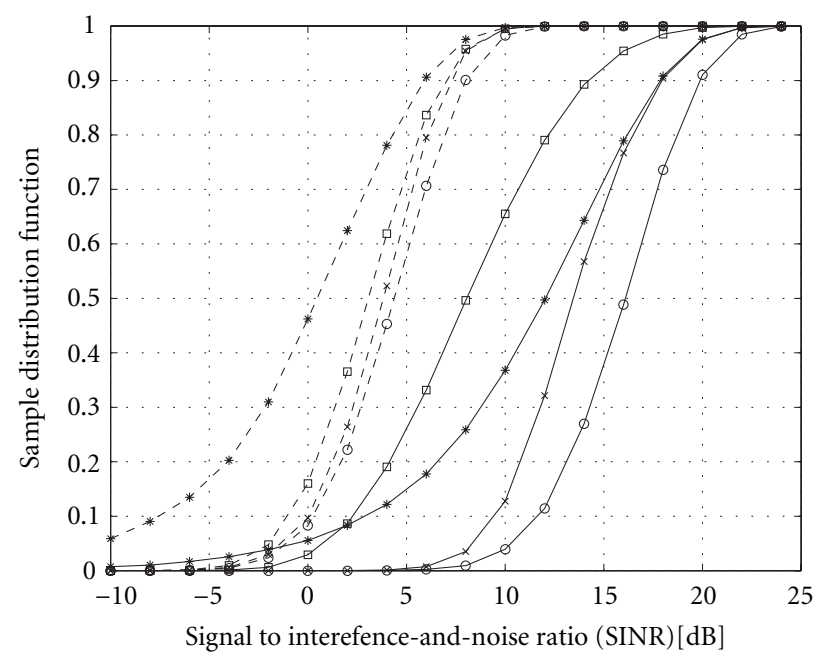

FIGURE 5: Sample distribution functions of SINR are presented at $6 \mathrm{~dB}$ (dashed line) and $18 \mathrm{~dB}$ (solid line) signal-to-noise ratios for Rake receiver $(\square)$, zero-forcing equalizer $(*)$, as well as for the LMMSE receivers given by $(7)(\circ)$ and by $(11)(\times)$.

In Figure 6, the average and the 10th percentile of SINR are presented with respect to $E_{S} / N_{0}$. As we could expect from Figure 5, the average SINR is not an adequate figure of merit. According to average SINR, the performance of the ZF equalizer approaches the performance of LMMSE equalizers with high $E_{S} / N_{0}$. However, the 10th percentile of SINR appears to be able to capture several performance characteristics. It shows that the performance of the Rake receiver saturates after $E_{S} / N_{0}=13 \mathrm{~dB}\left(E_{b} / N_{0}=10 \mathrm{~dB}\right)$, and that the performances of the $\mathrm{ZF}$ equalizer and Rake receiver cross at $E_{S} / N_{0}=18 \mathrm{~dB}$. It also shows that the performance of the LMMSE equalizer $\tilde{\mathbf{W}}_{L}$ starts to saturate at $E_{S} / N_{0}=23 \mathrm{~dB}$.

The ratio of symbol energy $E_{s}$ to the total power $P_{T}$ of base station signal was varied by scaling the powers of interfering users. The ratio $E_{s} / P_{T}$ describes the load of a base station, with low $E_{s} / P_{T}$ values indicating a heavily loaded system. At the same time, the $E_{s} / N_{0}$ values that maintained the 10th percentile of SINR at a predefined target value were searched. The target values were set to $-3 \mathrm{~dB}$ and $0 \mathrm{~dB}$, which resulted in all cases $13-15 \%$ and $6-8 \%$ BEPs, respectively. The results are presented in Figure 7. The horizontal differences between the curves indicate how much the transmission powers of interfering users can be increased while maintaining the desired user's transmission power constant. The amount that the desired user's transmission power can be decreased while maintaining the transmission powers of interfering users at a constant level is also indicated by the differences between the curves, as depicted in the figure.

\subsection{Bit error rate simulations}

The performance of adaptive chip-level equalizers were evaluated by simulations in Rayleigh fading Channel II defined in Table 2. In the simulations, the terminal speed was assumed to be $60 \mathrm{~km} / \mathrm{h}$, which results in a $56 \mathrm{~Hz}$ maximum 


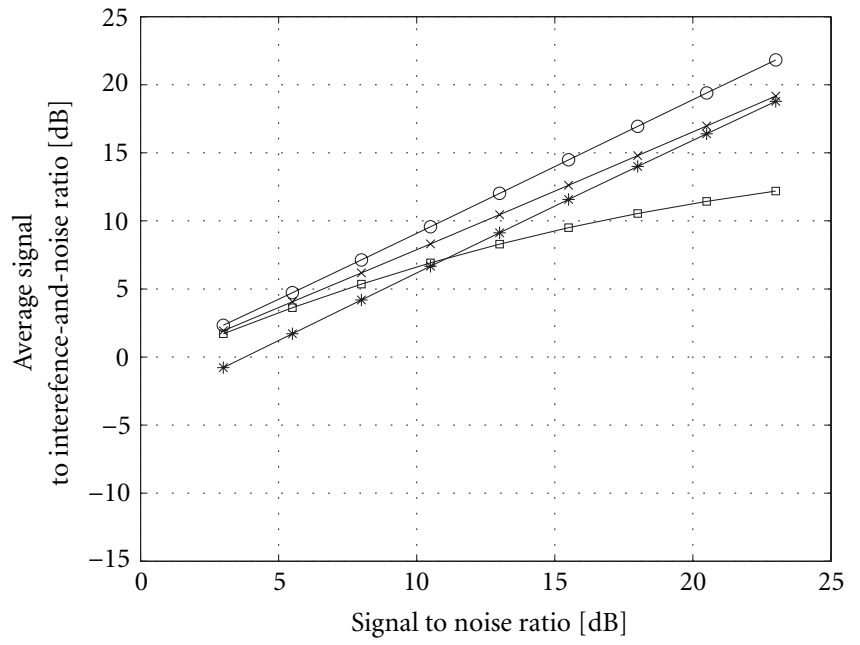

(a)

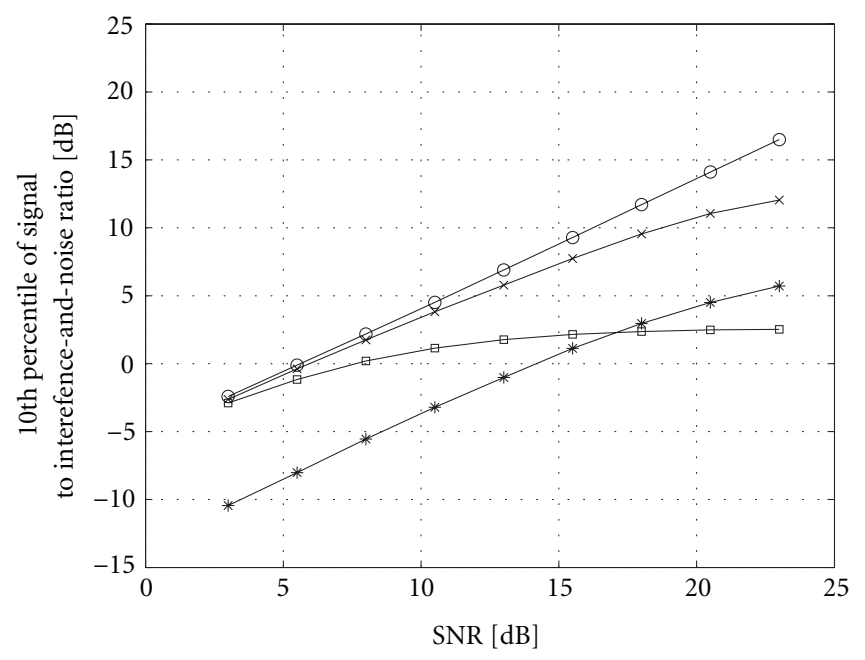

(b)

FIGURE 6: Average (a) and 10th percentile (b) of SINR versus signalto-noise ratio are presented for Rake receiver $(\square)$, zero-forcing equalizer $(*)$, as well as for the LMMSE receivers given by $(7)(\circ)$ and by $(11)(\times)$.

Doppler frequency shift at the $2 \mathrm{GHz}$ carrier frequency. A spreading factor of 64 was used on the common pilot channel, and the power of the pilot channel was scaled to $10 \%$ of the total transmitted power $P_{T}$. Filter lengths of 19 taps were used with the equalizers, except for the 16-tap CPICH trained equalizer.

BERs for different $E_{b} / N_{0}$ values are presented in Figure 8 for the considered adaptive receivers, excluding the $\mathrm{CPICH}$ trained equalizer. Also the performance of the LMMSE equalizer $\tilde{\mathbf{W}}_{L}$ and the theoretical single-user bound [49] for the considered channel are included in the figure. In Figure 8, all receivers had a perfect knowledge of the channel. The performance of the Rake receiver is degraded by MAI even at low

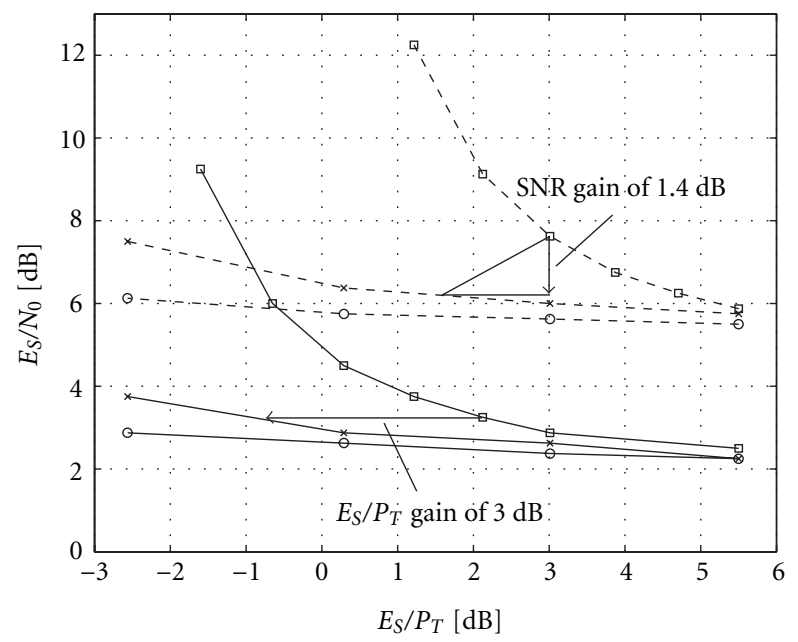

Figure 7: Required $E_{s} / N_{0}$ versus $E_{s} / P_{T}$ is presented for $-3 \mathrm{~dB}$ (solid line) and $0 \mathrm{~dB}$ (dashed line) target values of 10th percentile of SINR with Rake receiver $(\square)$ and LMMSE receivers given by (7) (०) and by $(11)(\times)$.

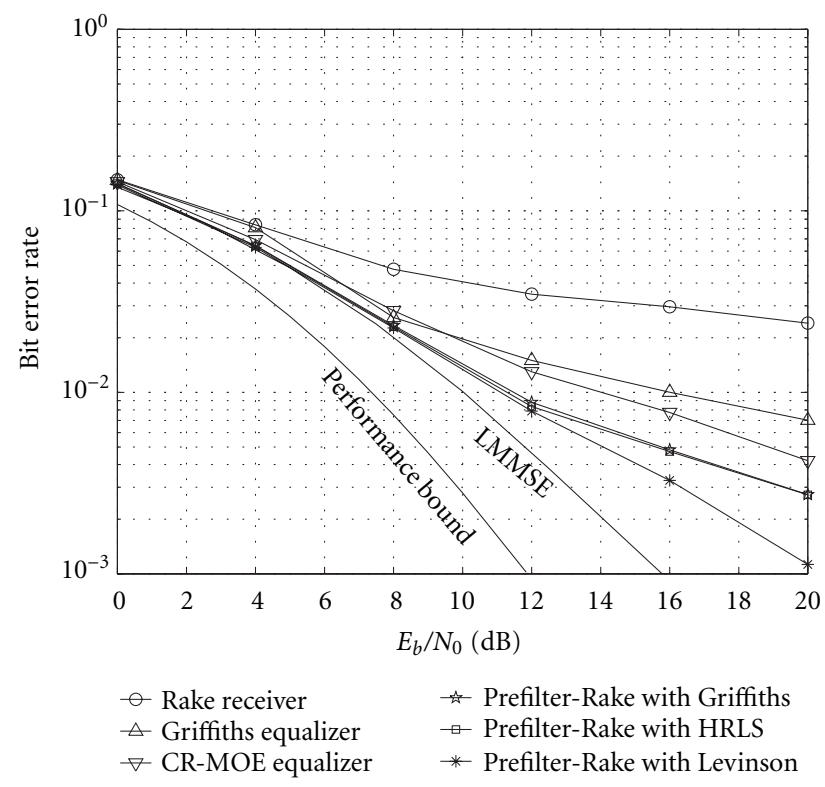

FIGURE 8: Bit error rates versus $E_{b} / N_{0}$ for 4 QPSK users (spreading factor 8) and common pilot channel (spreading factor 64) with known channel response in Channel II.

$E_{b} / N_{0}$ values, whereas the adaptive equalizers attain almost the performance of the LMMSE receiver at low $E_{b} / N_{0}$ values. At high $E_{b} / N_{0}$ values the prefilter-Rake receivers provide better performance than CR-MOE or Griffiths' equalizers. However, the performance differences remain at a moderate level.

To see the effect of channel estimation, the channel coefficients were estimated with a common pilot channel and a moving average smoother spanning over two slots. BERs are presented in Figure 9 for different $E_{b} / N_{0}$ values and in 


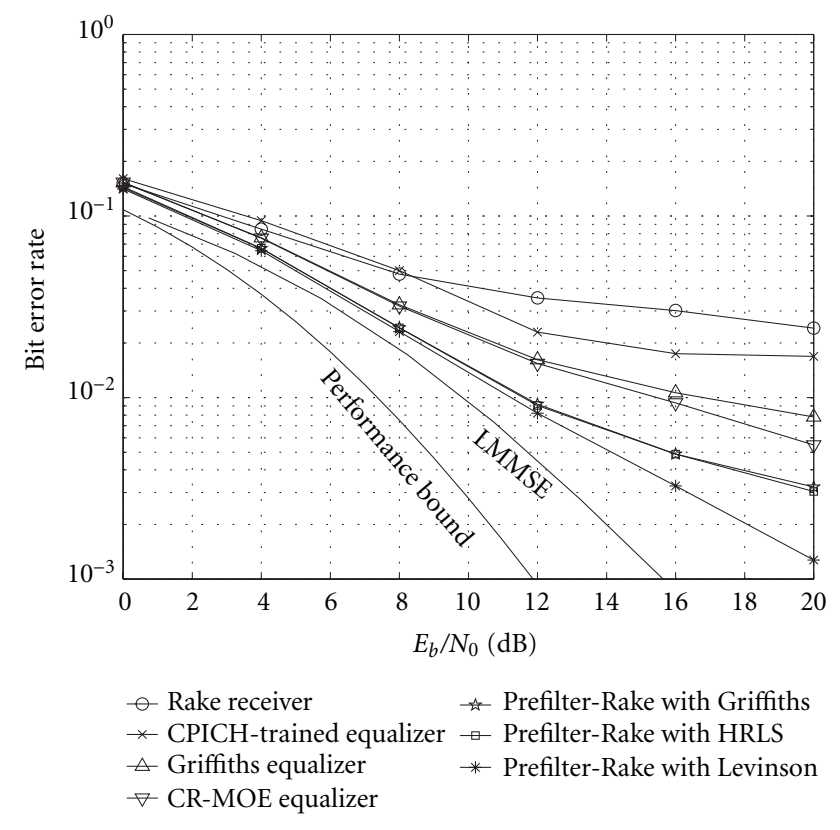

FIgURE 9: Bit error rates versus $E_{b} / N_{0}$ for 4 QPSK users (spreading factor 8) and common pilot channel (spreading factor 64) with estimated channel response in Channel II.

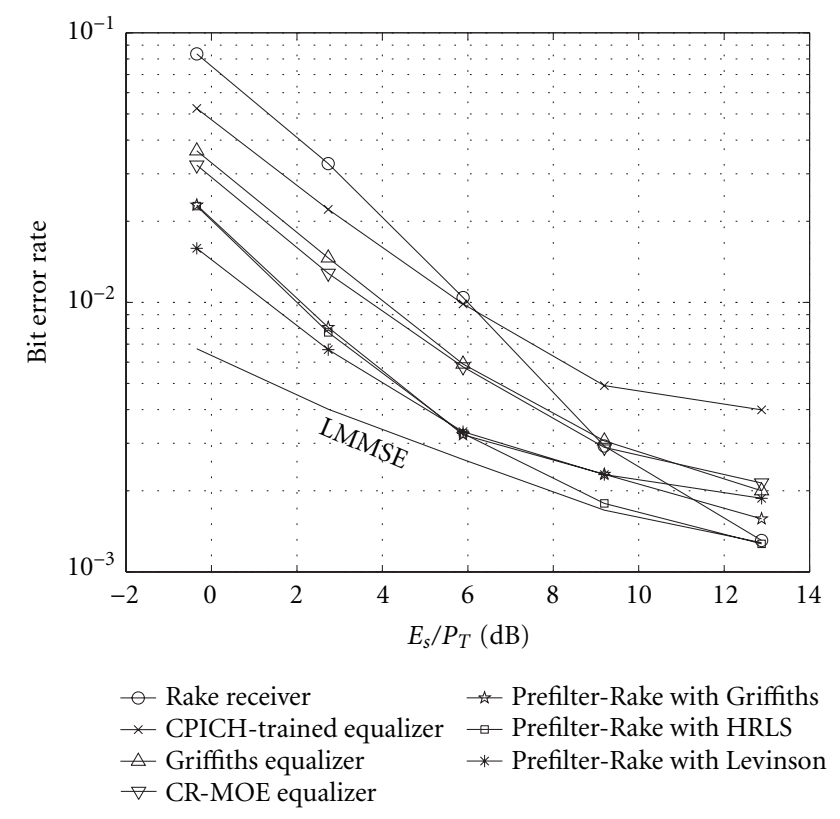

Figure 10: Bit error rates versus $E_{s} / P_{T}$ at $E_{b} / N_{0}=12 \mathrm{~dB}\left(E_{s} / N_{0}=\right.$ $15 \mathrm{~dB}$ ) with estimated channel response in Channel II.

Figure 10 with respect to the ratio of the desired user's symbol energy $E_{s}$ to the total transmitted power $P_{T}$. Different $E_{s} / P_{T}$ values were obtained by simulating different numbers of equal power users employing a spreading factor 64 .

Comparing Figures 8 and 9, it can be noted that the per- formance of a CR-MOE equalizer is affected by channel estimation errors. However, the performance degradation due to channel estimation is not severe. It is slightly surprising to see that the prefilter-Rake and Rake receivers are not significantly affected by channel estimation. This indicates that their performances are limited by other factors, like MAI in the case of the Rake receiver.

From the results it can be seen that both prefilterRake receivers as well as CR-MOE and Griffiths' equalizers provide performance improvements when compared to the conventional Rake receiver. The performance gain also increases with increasing $E_{b} / N_{0}$ (Figure 9) and decreasing $E_{s} / P_{T}$ (Figure 10), that is, when MAI becomes more dominant. However, at high $E_{s} / P_{T}$ values (low number of users) the Rake receiver provides equal or better performance than the CR-MOE or Griffiths' equalizer. From Figure 10 it can be noted that with decreasing numbers of users and, thus, MAI, the performance of prefilter-Rake and Rake receivers approach the performance of the LMMSE receiver.

The CPICH trained equalizer provides performance improvement over the Rake receiver at a relatively high $E_{b} / N_{0}$ range (Figure 9) or in severe MAI situations (Figure 10). The $\mathrm{CPICH}$ trained equalizer suffers from insufficient adaptation caused by low SNR in the adaptation. The SNR is especially low at high $E_{s} / P_{T}$ values, indicating low $\mathrm{CPICH}$ power. At the same range also the BER of the equalizer saturates as seen in Figure 10.

\section{CONCLUSIONS}

One approach to improve the performance of WCDMA downlink receivers was studied in this paper, namely, channel equalization prior to despreading. The presented receivers, consisting of a channel equalizer, a correlator, and a decision device, restore to some extent the orthogonality of users, and, thus, suppress MAI when orthogonal spreading sequences are employed.

The zero-forcing and LMMSE solutions for chip-level channel equalizers were defined and the effects of WCDMA downlink signal structure to the equalizers were addressed. The performance of chip-level channel equalizers was studied with respect to bit error probability and signal-tointerference-plus-noise ratio in a frequency-selective fading channel.

An overview of several adaptation methods proposed for the chip-level channel equalizers was given. Adaptive chiplevel channel equalizers based on training with a common pilot channel, on MOE equalization constrained with channel response, on the Griffiths' algorithm, as well as on the combination of a blind prefilter and Rake were studied. The performance of the equalizers was numerically evaluated and compared to the performance of the Rake receiver in a Rayleigh fading multipath channel. The results show significant performance improvements when chip-level channel equalizers are employed instead of the conventional Rake receiver in a WCDMA downlink. The prefilter-Rake receivers appear as the most promising adaptive solution for the equalizers. 


\section{ACKNOWLEDGMENTS}

The research at the University of Oulu has been supported by the Academy of Finland, Nokia, and Texas Instruments.

\section{REFERENCES}

[1] H. Holma and A. Toskala, Eds., Wideband CDMA for UMTS, John Wiley and Sons, New York, NY, USA, 2000.

[2] S. Verdú, Multiuser Detection, Cambridge University Press, Cambridge, UK, 1998.

[3] P. B. Rapajic and B. S. Vucetic, "Adaptive receiver structures for asynchronous CDMA systems," IEEE Journal on Selected Areas in Communications, vol. 12, no. 4, pp. 685-697, 1994.

[4] U. Madhow and M. L. Honig, "MMSE interference suppression for direct-sequence spread-spectrum CDMA," IEEE Trans. Communications, vol. 42, no. 12, pp. 3178-3188, 1994.

[5] A. Klein, Multi-user detection of CDMA signals_algorithms and their application to cellular mobile radio, Ph.D. thesis, University of Kaiserslautern, Kaiserslautern, Germany, 1996.

[6] A. Klein, "Data detection algorithms specially designed for the downlink of CDMA mobile radio systems," in Proc. IEEE Vehicular Tech. Conf., vol. 1, pp. 203-207, Phoenix, Ariz, USA, May 1997.

[7] C. D. Frank and E. Visotsky, "Adaptive interference suppression for direct-sequence CDMA systems with long spreading codes," in Proc. 36th Annual Allerton Conf. on Commun., Control, and Computing, pp. 411-420, Monticello, Ill, USA, September 1998.

[8] I. Ghauri and D. T. M. Slock, "Linear receivers for the DSCDMA downlink exploiting orthogonality of spreading sequences," in Proc. 32nd Asilomar Conf. on Signals, Systems, and Computers, vol. 1, pp. 650-654, Asilomar, Calif, USA, November 1998.

[9] K. Hooli, M. Latva-aho, and M. Juntti, "Multiple access interference suppression with linear chip equalizers in WCDMA downlink receivers," in Proc. IEEE Global Telecommunications Conf., vol. 1, pp. 467-471, Rio de Janeiro, Brazil, December 1999.

[10] M. Juntti and K. Hooli, "Overview on linear multiuser equalizers for DS-CDMA systems," in Wireless Communication Technologies: New Multimedia Systems, R. Kohno, N. Morinaga, and S. Sampei, Eds., chapter 5, pp. 97-129, Kluwer Academic, 2000.

[11] M. Bossert and T. Frey, "Interference cancellation in the synchronous downlink of CDMA systems," in Cost 231 TD(94)70, Prag, Czech, April 1994.

[12] T. Krauss and M. Zoltowski, "MMSE equalization under conditions of soft hand-off," in Proc. IEEE Int. Symp. Spread Spectrum Techniques and Applications, vol. 2, pp. 540-544, Parsippany, NJ, USA, September 2000.

[13] T. Krauss and M. Zoltowski, "Chip-level MMSE equalization at the edge of the cell," in Proc. IEEE Wireless Commun. and Networking Conf., vol. 1, pp. 386-392, Chicago, Ill, USA, September 2000.

[14] C. D. Frank, "MMSE reception of DS-CDMA with open-loop transmit diversity," in Proc. IEE Int. Conf. 3 G Mobile Commun. Tech., London, UK, March 2001.

[15] M. Lenardi, A. Medles, and D. Slock, "Comparison of downlink transmit diversity schemes for RAKE and SINR maximizing receivers," in Proc. IEEE Int. Conf. Commun., Helsinki, Finland, June 2001.
[16] P. Darwood, P. Alexander, and I. Oppermann, "LMMSE chip equalisation for 3GPP WCDMA downlink receivers with channel coding," in Proc. IEEE Int. Conf. Commun., Helsinki, Finland, June 2001.

[17] H. Elders-Boll, "Performance of an adaptive LMMSE chip equalizer for UTRA-FDD downlink detection," in Proc. COST 262 Workshop, pp. 1-6, Ulm, Germany, January 2001.

[18] M. Heikkilä, "A novel blind adaptive algorithm for channel equalization in WCDMA downlink," in Proc. IEEE Int. Symp. Pers., Indoor, Mobile Radio Commun., San Diego, Calif, USA, 30 September-3 October 2001.

[19] P. M. Grant, S. M. Spangenberg, D. G. M. Cruickshank, S. McLaughlin, and B. Mulgrew, "New adaptive multiuser detection technique for CDMA mobile receivers," in Proc. IEEE Int. Symp. Pers., Indoor, Mobile Radio Commun., vol. 1, pp. 52-54, Osaka, Japan, September 1999.

[20] S. Werner and J. Lilleberg, "Downlink channel decorrelation in CDMA systems with long codes," in Proc. IEEE Vehicular Tech. Conf., vol. 2, pp. 1614-1617, Houston, Tex, USA, July 1999.

[21] K. Li and H. Liu, "A new blind receiver for downlink DSCDMA communications," IEEE Communications Letters, vol. 3, no. 7, pp. 193-195, 1999.

[22] P. Komulainen and M. Heikkilä, "Adaptive channel equalization based on chip separation for CDMA downlink," in Proc. IEEE Int. Symp. Pers., Indoor, Mobile Radio Commun., vol. 3, pp. 1114-1118, Osaka, Japan, September 1999.

[23] M. Heikkilä, P. Komulainen, and J. Lilleberg, "Interference suppression in CDMA downlink through adaptive channel equalization," in Proc. IEEE Vehicular Tech. Conf., vol. 2, pp. 978-982, Amsterdam, The Netherlands, September 1999.

[24] P. Komulainen, M. Heikkilä, and J. Lilleberg, "Adaptive channel equalization and interference suppression for CDMA downlink," in Proc. IEEE Int. Symp. Spread Spectrum Techniques and Applications, vol. 2, pp. 363-367, Parsippany, NJ, USA, September 2000.

[25] S. Werner, M. L. R. de Campos, and J. A. Apolinario, "Kalman-filter based chip estimator for WCDMA downlink detection," in Proc. European Signal Processing Conf., Tampere, Finland, September 2000.

[26] K. Hooli, M. Latva-aho, and M. Juntti, "Novel adaptive channel equalizer for WCDMA downlink," in Proc. COST 262 Workshop, pp. 7-11, Ulm, Germany, January 2001.

[27] K. Hooli, M. Latva-aho, and M. Juntti, "Performance evaluation of adaptive chip-level channel equalizers in WCDMA downlink," in Proc. IEEE Int. Conf. Commun., vol. 6, pp. 19741979, Helsinki, Finland, June 2001.

[28] G. Bottomley, T. Ottoson, and Y.-P. E. Wang, "A generalized RAKE receiver for interference suppression," IEEE Journal on Selected Areas in Communications, vol. 18, no. 8, pp. 15361545, 2000.

[29] D. Dahlhaus and A. Jarosch, "Comparison of conventional and adaptive receiver concepts for the UTRA downlink," in Proc. Universal Mobile Telecommunications System (UMTS) Workshop, pp. 233-242, Reisensburg, Germany, November 1998.

[30] S. Qureshi, "Adaptive equalization," Proceedings of the IEEE, vol. 73, no. 9, pp. 1349-1387, 1985.

[31] D. P. Taylor, G. M. Vitetta, B. D. Hart, and A. Mämmelä, "Wireless channel equalization," European Transactions on Telecommunicatioms, vol. 9, no. 2, pp. 117-143, 1998. 
[32] S. M. Kay, Fundamentals of Statistical Signal Processing: Estimation Theory, Prentice-Hall, Englewood Cliffs, NJ, USA, 1993.

[33] K. Hooli, M. Latva-aho, and M. Juntti, "Comparison of LMMSE receivers in DS-CDMA downlink," in Proc. ACTS Mobile Communication Summit, vol. 2, pp. 649-654, Sorrento, Italy, June 1999.

[34] T. Krauss, W. Hillery, and M. Zoltowski, "MMSE equalization for forward link in 3G CDMA: Symbol-level versus chiplevel," in Proc. 10th IEEE Workshop on Statistical Signal and Array Processing, pp. 18-22, Pocono Manor, Pa, USA, August 2000.

[35] H. Liu, Signal Processing Applications in CDMA Communications, Artech House, Norwood, Mass, USA, 2000.

[36] D. Slock and I. Ghauri, "Blind maximum SINR receiver for the DS-CDMA downlink," in Proc. IEEE Int. Conf. Acoustics, Speech, Signal Processing, pp. 2485-2488, Istanbul, Turkey, June 2000.

[37] S. Mudulodu and A. Paulraj, "A blind multiuser receiver for the CDMA downlink," in Proc. IEEE Int. Conf. Acoustics, Speech, Signal Processing, pp. 2933-2936, Istanbul, Turkey, June 2000.

[38] S. Chowdhury, M. D. Zoltowski, and J. S. Goldstein, "Reduced-rank adaptive MMSE equalization for high-speed CDMA forward link with sparse multipath channels," in Conf. Record of the 34th Asilomar IEEE Conf. on Signals, Systems, and Computers, pp. 965-969, Pacific Grove, Calif, USA, 29 October-1 November 2000.

[39] F. Petre, M. Moonen, M. Engels, B. Gyselinckx, and H. De Man, "Pilot-aided adaptive chip equalizer receiver for intereference suppression in DS-CDMA forward link," in Proc. IEEE Vehicular Tech. Conf., vol. 1, pp. 303-308, Boston, Mass, USA, September 2000.

[40] J. R. Treichler, C. R. Johnson, and M. G. Larimore, Theory and Design of Adaptive Filters, John Wiley and Sons, New York, NY, USA, 1987.

[41] S. Haykin, Adaptive Filter Theory, Prentice-Hall, Upper Saddle River, NJ, USA, 3rd edition, 1996.

[42] M. Honig, U. Madhow, and S. Verdú, "Blind adaptive multiuser detection," IEEE Transactions on Information Theory, vol. 41, no. 3, pp. 944-960, 1995.

[43] L. Mailaender, "Low-complexity implementation of CDMA downlink equalization," in Proc. IEE Int. Conf. $3 G$ Mobile Commun. Tech., London, UK, March 2001.

[44] A. Rontogiannis and S. Theodoridis, "An adaptive LS algorithm based on orthogonal Householder transformations," in Proc. IEEE Int. Conf. on Electronics, Circuits, and Systems, pp. 860-863, 1996.

[45] T. K. Moon and W. C. Stirling, Mathematical Methods and Algorithms for Signal Processing, Prentice-Hall, Upper Saddle River, NJ, USA, 2000.

[46] G. H. Golub and C. F. Van Loan, Matrix Computations, The Johns Hopkins University Press, Baltimore, Md, USA, 2nd edition, 1989.

[47] ETSI, "Selection procedures for the choice of radio transmission technologies of the universal mobile telecommunications systems (UMTS)," Tech. Rep. version 3.0.0, European Telecommunications Standards Institute (ETSI), May 1997.

[48] M. J. Juntti and M. Latva-aho, "Bit error probability analysis of linear receivers for CDMA systems in frequency-selective fading channels," IEEE Trans. Communications, vol. 47, no. 12, pp. 1788-1791, 1999.
[49] M. K. Simon and M.-S. Alouini, "A unified approach to the performance analysis of digital communication over generalized fading channels," Proceedings of the IEEE, vol. 86, no. 9, pp. 1860-1877, 1985.

[50] J. G. Proakis, Digital Communications, McGraw-Hill, New York, NY, USA, 3rd edition, 1995.

Kari Hooli received his M.S. degree in electrical engineering from the University of Oulu, Finland in 1998. Since 1998, he has been a Research Scientist at Centre for Wireless Communications, University of Oulu. Currently, he is pursuing Dr.Tech. degree in electronic engineering with reseach interests in statistical signal processing, interference suppression, and CDMA systems.

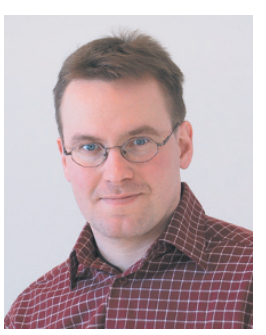

Markku Juntti was born in Kemi, Finland, in 1969. He received his M.S. (Tech.) and Dr.Tech. degrees in electrical engineering from University of Oulu, Oulu, Finland in 1993 and 1997, respectively. Dr. Juntti has been a Research Scientist and Research Project Manager at Telecommunication Laboratory and Centre for Wireless Communications, University of Oulu in 1992-1997. In academic year 1994-1995 he

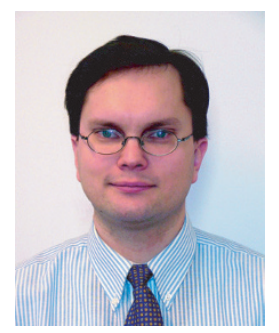
was a Visiting Research Scientist at Rice University, Houston, Tex. In 1998 he was an Acting Professor at the University of Oulu. In 1999-2000 he was with Nokia Networks, Radio Access Systems in Oulu as a Senior Specialist of W-CDMA Research and Solutions. Dr. Juntti has been a Professor of Telecommunications at University of Oulu since 2000. He is also Research Manager of UMTS Research at Centre for Wireless Communications, University of Oulu. Dr. Juntti consults the telecommunication industry, for example, by training its personnel. Dr. Juntti's research interests include communication theory and signal processing for wireless communication systems as well as their application in wireless communication system design. He is an author in book "W-CDMA for UMTS" published by Wiley. Dr. Juntti is a member of IEEE. He was Secretary of IEEE Communication Society Finland Chapter in 19961997 and the Chairman for years 2000-2001. He has been Secretary of the Technical Program Committee of the 2001 IEEE International Conference on Communications (ICC '01), and Chairman of the Technical Program Committees of 1999 Finnish Signal Processing Symposium (FINSIG '99) and the 2000 Finnish Wireless Communications Workshop (FWCW'00).

Markku J. Heikkilä received his M.S. degree in electrical engineering from the University of Oulu, Oulu, Finland, in 1997. He is currently a Senior Research Engineer in Nokia, Research and Technology Access, Finland. From 1996 to 1997, he was employed as a Research Assistant at the Telecommunication Laboratory of the University of Oulu. Since 1997, he has been with Nokia, Finland. His research interests include multi-

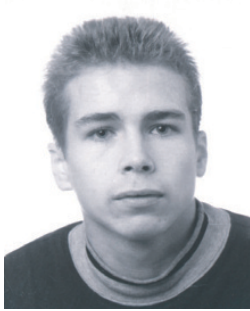
antenna transmission and reception techniques, space-time coding and baseband signal processing algorithms. 
Petri Komulainen was born in Kiuruvesi, Finland, in 1971. He received the M.S. (Dipl. Eng.) degree in 1996 from the programme of Information Engineering of the University of Oulu, Finland, with a thesis on turbo codes. In 1997, he worked as a Visiting Research Scholar at the New Jersey Institute of Technology (NJIT), Newark. Since then he has worked for Nokia Mobile Phones in Oulu, Finland, specialising in the

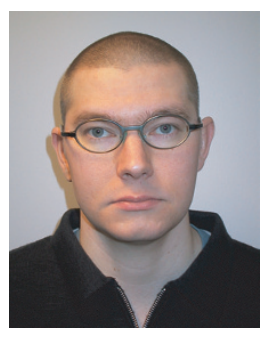
research and development of various WCDMA terminal receiver algorithms.

Matti Latva-aho received the M.S. in electrical engineering, Lic.Tech., and Dr.Tech. degrees from the University of Oulu, Finland in 1992, 1996, and 1998, respectively. From 1992 to 1993, he was a Research Engineer at Nokia Mobile Phones, Oulu, Finland. During the years 1994-1998 he was a Research Scientist at Telecommunication Laboratory and Centre for Wireless Communications at the University of Oulu. Cur-

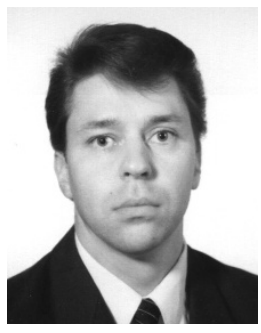
rently, Professor Latva-aho is Director of Centre for Wireless Communications at the University of Oulu. His research interests include future broadband wireless communication systems and related transceiver algorithms. Professor Latva-aho has published more than 50 conference or journal papers in the field of CDMA communications.

Jorma Lilleberg was born in Rovaniemi, Finland, in 1953. He received the Diploma Engineer and Licentiate of Technology degrees in electrical engineering at the University of Oulu, Oulu, Finland in 1979 and 1984, respectively and the Doctor of Technology degree at the Tampere University of Technology, Tampere, Finland in 1992. From 1992 to 1993 he worked at the Tech-

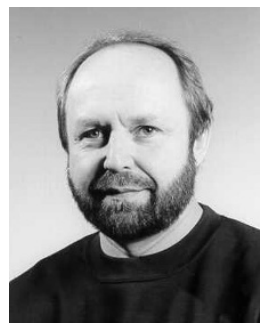
nical Research Center of Finland, in Oulu, Finland as an acting Research Professor and Chief Scientist. Since August 1993 he has been working at Nokia Mobile Phones, Oulu, Finland as a Principal Scientist and NMP Fellow. Dr. Lilleberg's research interest is in digital communications theory and the application of statistical signal processing methods to digital radio receivers. He has published several tens of research papers and holds 9 patents. He is also a Docent to the University of Oulu, Oulu, Finland where he has given lectures on estimation theory and guided doctoral students. 